\author{
В.Н. Кравец, Р.А. Мусарский, А.В. Тумасов
}

\title{
ВЛИЯНИЕ ПАРАМЕТРОВ ТРАНСМИССИИ НА ПОКАЗАТЕЛИ ТЯГОВО-СКОРОСТНЫХ СВОЙСТВ ГРУЗОВОГО АВТОМОБИЛЯ
}

\author{
Нижегородский государственный технический университет им. Р.Е. Алексеева
}

\begin{abstract}
Представлена конструкция двигателя модернизированной модели ГАЗон Next 10, более мощная по сравнению с существующими аналогами: пятиступенчатая коробка передач заменяется на шестиступенчатую, предполагается установка двух вариантов главной передачи с передаточными числами 3,9 и 4,55. Изменение параметров двигателя и трансмиссии оказало влияние на показатели модели ГАЗон Next 10. В качестве главных задач исследования определены разработка методики определения показателей тягово-скоростных свойств грузового автомобиля и установление зависимостей между величинами конструктивных параметров трансмиссии и показателями тягово-скоростных свойств исследуемого автомобиля. Определение названных показателей выполнено на основе решения графическим методом уравнения прямолинейного движения автомобиля с построением графиков силового и мощностного балансов, динамической характеристики, ускорений, времени и пути разгона. Установлено, что снижение передаточного числа главной передачи с 4,55 до 3,9 (на 17 \%) повысило максимальную скорость автомобиля с пятиступенчатой коробкой передач на 16\%, автомобиля с шестиступенчатой коробкой передач - на 10\%; с увеличением передаточного числа главной передачи с 3,9 до 4,55 максимальный уклон дороги, преодолеваемый автомобилем, линейно возрастает с 31,8 \% до 37 \%, а максимальные ускорения разгона увеличиваются на всех передачах коробки передач: на I - на 2,3 \%, на II - на 10,2 \%, на III - на 17 \%, на IV - на 19,8 \%, на V - на 26 \%, на VI - на 40 \%; удельная мощность автомобиля 12,5 кВт/т. Проведенные исследования позволяют более обоснованно подойти к выбору параметров трансмиссии модернизируемого грузового автомобиля ГАЗон Next 10.
\end{abstract}

Ключевые слова: грузовой автомобиль, трансмиссия автомобиля, механическая ступенчатая коробка передач, главная передача, тягово-скоростные свойства автомобиля, показатели тягово-скоростных свойств.

\section{Обоснование необходимости и цели работ}

В сентябре 2014 г. Группа ГАЗ в Нижнем Новгороде приступила к серийному производству семейства среднетоннажных грузовых автомобилей пятого поколения ГАЗон Next грузоподъемностью 5 т и полной массой 8,7 т. Семейство состоит из трех групп: универсальной, городской и внедорожной. На автомобилях универсальной группы, пришедшим на замену модели ГАЗ-3307, установлены колеса с камерными шинами 8.25R20. На автомобилях городской группы с целью снижения погрузочной высоты платформы предусмотрена установка колёс с низкопрофильными бескамерными шинами 265/70R19,5. Группа внедорожных автомобилей с колесной формулой $4 \times 4$, оснащенных шинами повышенной проходимости, пришла на смену армейскому автомобилю ГАЗ-3308 «Садко». На всех моделях семейства ГАЗон Next был установлен отечественный дизельный двигатель с турбонаддувом ЯМ3-5344 максимальной мощностью 109,5 кВт (148,9 л.с.) и модернизированная пятиступенчатая коробка передач, способная передавать к ведущим колесам крутящий момент до $500 \mathrm{H} \cdot$ м.

С учетом трехлетнего опыта эксплуатации семейства автомобилей ГАЗон Next предприятие в 2018 г. приступило к разработке более совершенной модели ГАЗон Next 10. С целью повышения производительности и снижения себестоимости перевозок грузоподъемность модернизированной модели увеличена до 6,2 т, в результате чего полная масса возросла до 10 т. Для обеспечения движения автомобиля с максимальной скоростью до 105 км/ч потребовалась установка дизельного двигателя ЯМЗ-53445 максимальной мощностью 125 кВт (170 л.с.) и максимальным крутящим моментом $650 \mathrm{H} \cdot$ м. Предполагается замена пятиступенчатой коробки передач на шестиступенчатую с шестой повышающей ступенью и установка двух вариантов главной передачи с передаточными числами 3,9 и 4,55. На неполнопроводных моделях

(C) Кравец В.Н., Мусарский Р.А., Тумасов А.В. 
предусмотрена установка низкопрофильных шин 265/70R19,5. Изменение параметров двигателя, трансмиссии и шин неизбежно должно оказать влияние на показатели тягово-скоростных свойств модернизированной модели. Соответственно, основными задачами исследования были определены:

1) разработка методики определения показателей тягово-скоростных свойств исследуемого грузового автомобиля;

2) установление зависимостей между величинами конструктивных параметров трансмиссии и показателями тягово-скоростных свойств исследуемого автомобиля.

Исследования выполнены при финансовой поддержке Министерства образования и науки РФ в рамках проекта «Создание высокотехнологичного производства безопасных экспортоориентированных автомобилей ГАЗ с элементами автономного управления и возможностью интеграции с электроплатформой на базовых компонентах российского производства» по договору №03.G25.31.0270 от 29.05.2017г. (постановление Правительства Российской Федерации от 09 апреля 2010 года №218).

\section{Методика выполнения работы}

В качестве объекта исследования принят грузовой автомобиль ГАЗон Next 10, являющийся, согласно международной и отечественной классификации, транспортным средством категории $\mathrm{N}_{2}$. Для проведения исследования были использованы исходные данные, предоставленные конструкторами Группы ГАЗ:

- полная масса $m_{\mathrm{a}}=10000$ кг;

- двигатель дизельный с турбонаддувом ЯМЗ-53445-20, максимальная стендовая мощность $P_{e \max }^{\mathrm{c}}=125$ кВт (170 л.с.) при частоте вращения $n_{e P}=2300$ об/мин, максимальный стендовый крутящий момент двигателя $T_{e \max }^{\mathrm{c}}=650 \mathrm{H} \cdot \mathrm{M}$ при частоте $n_{e T}$ $=1200 \ldots 1600$ об/мин;

- коэффициент коррекции стендовой характеристики двигателя $k_{\mathrm{c}}=0,95$;

- коробка передач механическая ступенчатая с двумя вариантами передаточных чисел:

вариант 1 - пятиступенчатая с передаточныли числами $u_{к \mathrm{I}}=6,555 ; \quad u_{\kappa \mathrm{II}}=3,933 ; \quad u_{\kappa \mathrm{III}}$ $=2,376 ; \quad u_{\kappa \mathrm{IV}}=1,442 ; \quad u_{\kappa \mathrm{V}}=1,000 ;$

вариант 2 - шестиступенчатая с передаточныли числами $u_{\kappa \mathrm{I}}=6,555 ; \quad u_{\kappa \mathrm{II}}=3,933$; $u_{\kappa \mathrm{III}}=2,376 ; \quad u_{\kappa \mathrm{IV}}=1,442 ; \quad u_{\kappa \mathrm{V}}=1,000 ; \quad u_{\kappa \mathrm{VI}}=0,782 ;$

- главная передача с двумя вариантами передаточных чисел: вариант $1-u_{0}=3,9 ;$ вариант $2-u_{0}=4,55$.

- коэффициент полезного действия трансмиссии $\eta_{\mathrm{TP}}=0,87$;

- шины бескамерные низкопрофильные 265/70R19,5, статический радиус колес $r_{\text {ст }}=0,421 \mathrm{м}$, динамический радиус колес $r_{\text {д }}=0,421 \mathrm{м}$, радиус качения колёс $r_{\mathrm{k}}=$ $0,438 \mathrm{M}$;

- коэффициент сопротивления качению при скорости, близкой к нулю, $f_{0}=0.01$;

- коэффициент, учитывающий влияние скорости на потери при качении, $k_{f}=0,0001 \mathrm{c} / \mathrm{M}$;

- параметры обтекаемости: коэффициент обтекаемости $c_{x}=0,7$; плотность воздуха $\rho_{\mathrm{B}}=1,225$ кг $/ \mathrm{m}^{3}$; площадь миделя $A_{\mathrm{B}}=4,15 \mathrm{~m}^{2}$; фактор обтекаемости $W=1,78\left(\mathrm{H} \cdot \mathrm{c}^{2}\right) / \mathrm{m}^{2}$; 
- момент инерции вращающихся частей двигателя $J_{e}=2,15$ кг $\mathbf{M}^{2}$, колеса $J_{\text {к }}=9,36$ кг $\cdot \mathrm{M}^{2}$, число колес $n_{\mathrm{\kappa}}=6$.

Для оценки тягово-скоростных свойств автомобиля были использованы единичные показатели, содержащиеся в нормативных документах [1-5];

1) максимальная скорость $v_{\text {amax }}$;

2) время разгона на заданном пути 400 м и 1000 м $t_{400}$ и $t_{1000}$;

3) время разгона до заданной скорости 80 км/ч $t_{80}$;

4) максимальный преодолеваемый автомобилем уклон дороги $i \%$;

5) максимальные ускорения разгона автомобиля на низшей и высшей ступенях коробки передач $a_{\text {amax }}, a_{\text {amaxV }}, a_{\text {amaxVI }}$;

6) удельная мощность автомобиля $P_{\text {уд }}$.

Определение названных показателей выполнено на основе решения графическим методом уравнения прямолинейного движения автомобиля, изложенным в учебниках по теории автомобиля [6-9]. Были рассчитаны и построены графики: 1) силового баланса; 2) мощностного баланса: 3) динамической характеристики; 4) ускорений разгона; 5) времени разгона; 6) пути разгона с пяти- и шестиступенчатой коробками передач при дискретных значениях передаточных чисел главной передачи $u_{0}=3,9 ; 4,1 ; 4,3 ; 4,55$. На всех перечисленных графиках наносят численные значения скорости автомобиля (км/ч), которую рассчитывают по формуле (1):

$$
v_{\mathrm{a}}=\frac{0,378 n_{e} r_{\kappa}}{u_{\kappa} u_{\mathrm{A}} u_{0}},
$$

где $n_{e}$ - частота вращения вала двигателя, об/мин; $r_{\text {к }}$ радиус качения ведущих колес, м; $u_{\text {к }}$, $u_{д}, u_{0}$ - передаточные числа коробки передач, дополнительной и главной передач соответственно.

Силовой (тяговый) баланс автомобиля показывает, как распределяется полная тяговая сила на ведущих колёсах по отдельным видам сопротивления движению.

Уравнение силового баланса $(\mathrm{H})$ :

- в общем виде (2)

$$
F_{\mathrm{T}}=F_{f}+F_{i}+F_{\mathrm{B}}+F_{a}+F_{\mathrm{cx}} ;
$$

- в развернутом виде при отсутствии буксования ведущих колес (3)

$$
\frac{k_{c} T_{e}^{c} u_{\kappa} u_{\text {д }} u_{0} \eta_{\text {тр }}}{r_{\text {д }}}=f G_{\mathrm{a}} \cos \alpha+G_{\mathrm{a}} \sin \alpha+\frac{W v_{\mathrm{a}}^{2}}{12,96}+\delta m_{\mathrm{a}} a_{\mathrm{a}}+F_{\mathrm{c} x},
$$

где $F_{\text {т }}$ - тяговая сила на ведущих колёсах, $\mathrm{H} ; F_{f}$ - сила сопротивления качению, $\mathrm{H} ; F_{i}-$ сила сопротивления подъему, $\mathrm{H} ; F_{\text {в }}$ - сила сопротивления воздуха , $\mathrm{H} ; F_{a}$ - сила сопротивления разгону, $\mathrm{H} ; F_{c x}$ - сила сопротивления прицепа, $\mathrm{H} ; k_{c}$ - коэффициент коррекции стендовой характеристики двигателя; $T_{e}^{c}$ - эффективный стендовый крутящий момент двигателя, $\mathrm{H} \cdot \mathrm{M} ; u_{\kappa}, u_{\text {д }}$, $u_{0}$ - передаточные числа коробки передач, дополнительной и главной передач соответ-

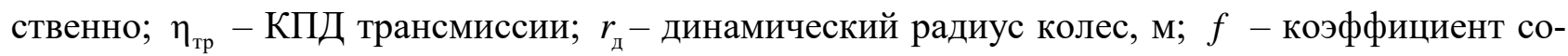
противления качению; $G_{\text {a }}$ вес автомобиля, $\mathrm{H} ; \alpha-$ угол продольного уклона дороги, рад или град; $W$ - фактор обтекаемости автомобиля, $\left(\mathrm{H} \cdot \mathrm{c}^{2}\right) / \mathrm{M}^{2} ; v_{\mathrm{a}}-$ скорость автомобиля, км/ч; $\delta$ коэффициент учета вращающихся масс; $m_{\mathrm{a}}$ - масса автомобиля, кг; $a_{\mathrm{a}}$ - ускорение разгона автомобиля, м/ $\mathrm{c}^{2}$. 
Мощностной баланс показывает, как распределяется тяговая мощность, подводимая к ведущим колесам автомобиля, по отдельным видам сопротивления движению.

Уравнение мощностного баланса (кВт):

- в общем виде (4)

$$
P_{\mathrm{T}}=P_{f}+P_{i}+P_{\mathrm{B}}+P_{a}+P_{\mathrm{c} x}
$$

- в развернутом виде (5)

$$
k_{c} P_{e}^{c} \eta_{\text {тр }}\left(1-s_{\sigma}\right)=\frac{f G_{\mathrm{a}} v_{\mathrm{a}} \cos \alpha+G_{\mathrm{a}} v_{\mathrm{a}} \sin \alpha+W v_{\mathrm{a}}^{3} / 12.96+\delta m_{\mathrm{a}} a_{\mathrm{a}} v_{\mathrm{a}}+F_{\mathrm{cx}} v_{\mathrm{a}}}{3600},
$$

где $P_{e}^{c}$ - эффективная стендовая мощность двигателя, кВт; $s_{\sigma}-$ коэффициент буксования ведущих колес.

Динамической характеристикой называют зависимость динамического фактора от скорости автомобиля на всех передачах трансмиссии. Динамический фактор автомобиля:

- в общем виде (6)

$$
D=\frac{F_{\mathrm{cB}}}{G_{\mathrm{a}}}=\frac{F_{\mathrm{T}}-F_{\mathrm{B}}}{m_{\mathrm{a}} g}
$$

- в развернутом виде (7)

$$
D=\frac{\frac{k_{c} T_{e}^{c} u_{\mathrm{r}} u_{\text {д }} u_{0} \eta_{\text {тр }}}{r_{\text {д }}}-\frac{W v_{\mathrm{a}}^{2}}{12,96}}{m_{\mathrm{a}} g}
$$

График ускорений представляет зависимость ускорений разгона от скорости автомобиля на всех передачах коробки передач. При движении автомобиля по горизонтальной по-

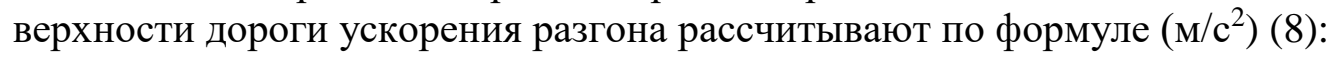

$$
a_{\mathrm{a}}=\frac{(D-f) g}{\delta},
$$

где $D$ - динамический фактор; $f$ - коэффициент сопротивления качению; $g$ - ускорение свободного падения, м/ $\mathrm{c}^{2} ; \delta$ - коэффициент учета вращающихся масс.

Время разгона автомобиля (c):

- $\quad$ в $i$-м интервале изменения скорости (9)

$$
\Delta t_{i}=\frac{\Delta v_{\mathrm{a} i}}{3.6 a_{\mathrm{aicp}}}=\frac{v_{\mathrm{a} i}-v_{\mathrm{a}(i-1)}}{1.8\left(a_{\mathrm{a}(i-1)}-a_{\mathrm{a} i}\right)},
$$

- на $j$-й передаче (10)

$$
t_{j}=\sum_{i=1}^{n} \Delta t_{i},
$$

- общее от минимальной до максимальной скорости (11):

$$
t=\sum_{j=1}^{m} \sum_{i=1}^{n} \Delta t_{i}+\sum_{j=1}^{m-1} t_{\Pi}
$$

где $\Delta v_{\text {аi }}-i$-й интервал скоростей, км/ч; $a_{\text {аiср }}$ - среднее ускорение в $i$-м интервале скоростей, $\mathrm{м} / \mathrm{c}^{2} ; v_{\mathrm{a}(i-1)}$ и $v_{\mathrm{a} i}-$ скорости в начале и конце $i$ - го интервала, км/ч; $i$ - порядковый номер интервала изменения скорости; $n$ - число интервалов скоростей на $j$ - й передаче; $m$ - число передач в коробке передач; $t_{\text {п }}$ - время переключения с одной передачи на другую.

Аналогично рассчитывают путь разгона автомобиля. В качестве примера на рис. 1 представлена динамическая характеристика автомобиля с шестиступенчатой коробкой передач и главной передачей с передаточным числом 3,9, на рис. 2 и 3 - графики времени и пути разгона 
автомобиля с шестиступенчатой коробкой передач при передаточных числах главной передачи 4,$55 ; 4,3 ; 4,1 ; 3,9$.

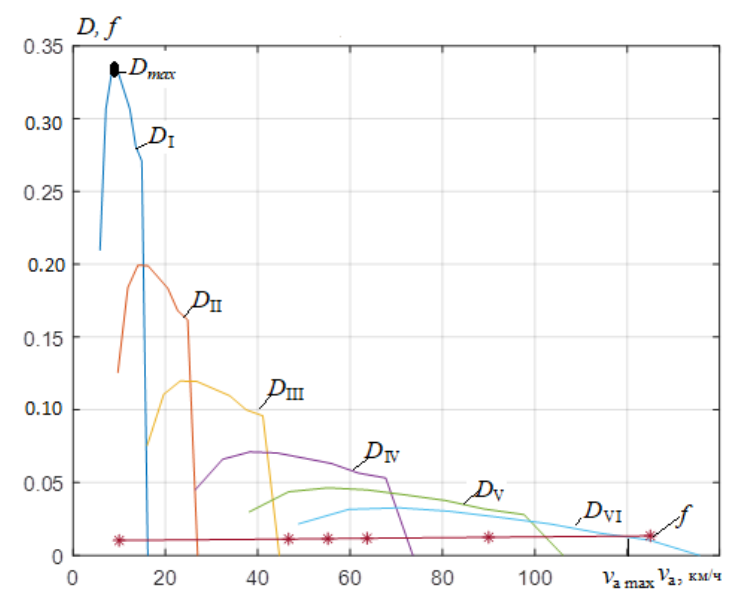

Рис. 1. Динамическая характеристика автомобиля с шестиступенчатой коробкой передач и главной передачей с передаточным числом 3,9

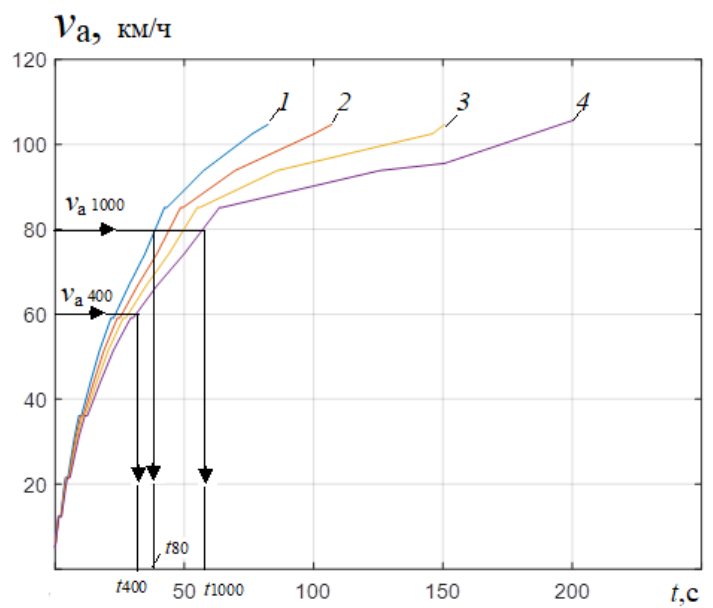

Рис. 2. Время разгона автомобиля с шестиступенчатой коробкой передач при передаточных числах главной передачи: 1 - 4,55; 2 - 4,3; 3 - 4,1; 4-3,9

По графику мощностного баланса в точке пересечения кривых тяговой мощности на ведущих колёсах $P_{\mathrm{T}}$ и суммы мощностей, затрачиваемых на преодоление сил сопротивления качению и воздуха, $P_{f}+P_{\text {в }}$.

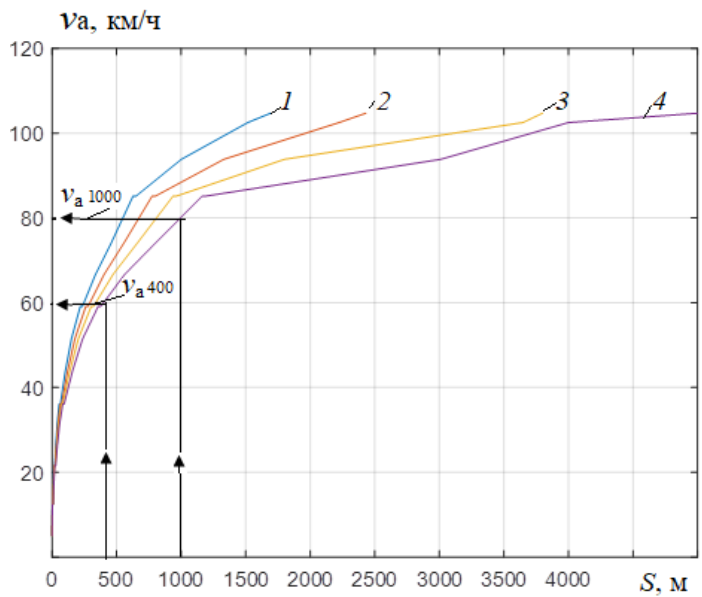

Рис. 3. Путь разгона автомобиля с шестиступенчатой коробкой передач при передаточных числах главной передачи: 1 - 4,55; 2 - 4,3; 3-4,1; 4-3,9 
Наличие 48 графиков позволило в полной мере выявить влияние параметров трансмиссии на перечисленные выше показатели тягово-скоростных свойств автомобиля ГАЗон Next 10. Максимальную скорость автомобиля определяют по одному из графиков:

- по графику силового баланса в точке пересечения кривых полной тяговой силы на ведущих колесах $F_{\mathrm{T}}$ и суммы сил сопротивления качению и воздуха $F_{f}+F_{\text {в }}$;

- по графику динамической характеристики (рис. 1) в точке пересечения кривых динамического фактора $D_{\mathrm{VI}}$ и коэффициента сопротивления качению $f$;

- по графику ускорений разгона в точке пересечения кривой ускорения с осью абсцисс.

Время разгона на заданном пути определяют на горизонтальном участке длиной 400 м и 1000 м $\left(t_{400}\right.$ и $\left.t_{1000}\right)$. Для их определения в начале по графику пути разгона (рис. 3$)$ находят скорости $v_{\text {a } 400}$ и $v_{\mathrm{a} 1000}$, достигаемые на этих отрезках пути, а затем по графику времени разгона (рис. 2) при этих скоростях искомые значения времени разгона $t_{400}$ и $t_{1000}$.

Для грузовых автомобилей полной массой свыше 3,5 т время разгона определяют до заданной скорости 80 км/ч ( $\left.t_{80}\right)$ по графику времени разгона (рис. 2).

Максимальный угол преодолеваемого автомобилем подъема (град) рассчитывают по формуле (12):

$$
\alpha_{\max }=\arcsin \left[\frac{D_{\text {max }}-f \sqrt{1-D_{\text {max }}^{2}+f^{2}}}{1+f^{2}}\right],
$$

где $D_{\max }$ - максимальный динамический фактор на низшей ступени коробки передач; $f$ - коэффициент сопротивления качению при скорости, соответствующей $D_{\max }$.

Численные значения $D_{\max }$ берут с графика динамической характеристики (рис. 1), коэффициент сопротивления качению принимают равным $f_{0}=0,01$. нию (13):

Максимальный уклон дороги (\%), преодолеваемый автомобилем, находят по выраже-

$$
i_{\max }=100 \operatorname{tg} \alpha_{\max }
$$

Максимальные ускорения разгона на низшей и высшей ступенях коробки передач определяют по графику ускорений разгона $a_{\mathrm{a}}=f\left(v_{\mathrm{a}}\right)$.

Удельную мощность автомобиля (кВт/т) находят по выражению:

$$
P_{\text {уд }}=\frac{P_{e \max }^{\mathrm{c}}}{m_{\mathrm{a}}},
$$

где $P_{e \text { max }}^{\mathrm{c}}-$ максимальная стендовая мощность двигателя, квт; $m_{\mathrm{a}}-$ полная масса автомобиля, т.

\section{Результаты исследования}

На рис. 4 представлена зависимость максимальной скорости автомобиля с пяти- и шестиступенчатой коробками передач от передаточного числа главной передачи. 


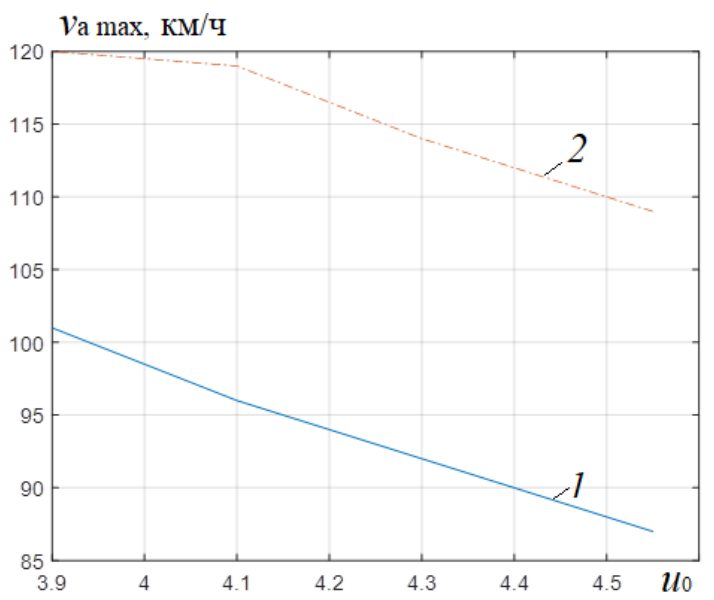

Рис. 4. Зависимость максимальной скорости автомобиля от передаточного числа главной передачи с коробками передач: 1 - пятиступенчатой; 2 - шестиступенчатой

В табл. 1 и 2 показаны зависимости времени разгона на пути 400 м и 1000 м и времени разгона до скорости 80 км/ч от параметров трансмиссии.

Таблица 1

Зависимость времени разгона на пути 400 м и 1000 м от параметров трансмиссии

\begin{tabular}{|c|c|c|c|c|c|}
\hline $\begin{array}{c}\text { Количество ступеней } \\
\text { в коробке передач }\end{array}$ & $\begin{array}{c}\text { Передаточное число } \\
\text { главной передачи }\end{array}$ & 4,55 & 4,3 & 4,1 & 3,9 \\
\hline \multirow{2}{*}{ Пять } & $\begin{array}{c}\text { Время разгона на пути } \\
400 \text { м, с }\end{array}$ & 32,5 & 32,5 & 32,5 & 32,5 \\
\cline { 2 - 6 } & $\begin{array}{c}\text { Время разгона на пути } \\
1000 \text { м, с }\end{array}$ & - & - & 63 & 63 \\
\hline \multirow{2}{*}{ Шесть } & $\begin{array}{c}\text { Время разгона на пути } \\
400 \text { м, с }\end{array}$ & 32 & 32 & 32 & - \\
\cline { 2 - 6 } & $\begin{array}{c}\text { Время разгона на пути } \\
1000 \text { м, с }\end{array}$ & 64 & 65 & 65 & - \\
\hline
\end{tabular}

Таблица 2

Зависимость времени разгона до скорости 80 км/ч от параметров трансмиссии

\begin{tabular}{|c|c|c|c|c|c|}
\hline $\begin{array}{c}\text { Количество ступеней } \\
\text { в коробке передач }\end{array}$ & $\begin{array}{c}\text { Передаточное число } \\
\text { главной передачи }\end{array}$ & 4,55 & 4,3 & 4,1 & 3,9 \\
\hline Пять & $\begin{array}{c}\text { Время разгона } \\
\text { до скорости } 80 \text { км/ч, с }\end{array}$ & 41 & 41,2 & 41 & 41 \\
\hline Шесть & $\begin{array}{c}\text { Время разгона } \\
\text { до скорости } 80 \text { км/ч, с }\end{array}$ & 39 & 40 & 41 & 41,5 \\
\hline
\end{tabular}

Зависимость максимального преодолеваемого автомобилем уклона дороги (\%) от передаточного числа главной передачи может быть аппроксимирована уравнением: $i=31,8+8,0\left(u_{0}-3,9\right)$.

Рис. 5 иллюстрирует влияние передаточного числа главной передачи на максимальные ускорения разгона на I ... VI ступенях коробки передач. 


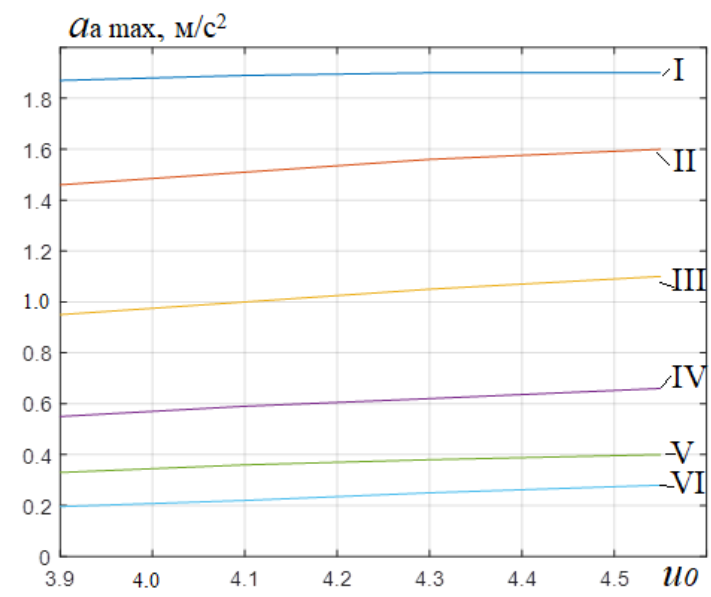

Рис. 5. Зависимость максимальных ускорений разгона от передаточного числа главной передачи на всех ступенях коробки передач

\section{Выводы}

1. При уменьшении передаточного числа главной передачи максимальная скорость автомобиля возрастает. Снижение передаточного числа главной передачи с 4,55 до 3,9 (на $17 \%$ ) повысило максимальную скорость автомобиля с пятиступенчатой коробкой передач с 87 км/ч до 101 км/ч (на 16 \%), автомобиля с шестиступенчатой коробкой передач - с 109 км/ч до 120 км/ч (на $10 \%)$.

2. Изменение передаточного числа главной передачи не оказывает влияния на время разгона автомобиля на пути 400 м и 1000 м и на время его разгона до скорости $80 \mathrm{\kappa м} /$ ч.

3. С увеличением передаточного числа главной передачи с 3,9 до 4,55 максимальный уклон дороги, преодолеваемый автомобилем, линейно возрастает с 31,8 \% до $37 \%$.

4. Повышение передаточного числа главной передачи с 3,9 до 4,55 приводит к увеличению максимальных ускорений разгона на всех ступенях шестиступенчатой коробки передач: на первой передаче на $2,3 \%$, на второй - на 10,2 \%, на третьей - на $16,7 \%$, на четвертой - на 19,2 \%, на пятой - на $26 \%$, на шестой - на $40 \%$.

5. Удельная мощность автомобиля 12,5 кВт/т.

6. Выполненное исследование позволит предприятию более обоснованно подойти к выбору параметров трансмиссии модернизируемой модели грузового автомобиля ГАЗон Next 10.

\section{Библиографический список}

1. Правила ЕЭК ООН № 68. Единообразные предписания, касающиеся официального утверждения автотранспортных средств в отношении измерения максимальной скорости. - Введ. 01.09.96. Швейцария, Женева: ЕЭК ООН, 1996. - 16 с.

2. ГОСТ 22576 - 90 (СТ СЭВ 6893 - 89). Автотранспортные средства. Скоростные свойства. Методы испытаний. - Взамен ГОСТ 22576 - 77; введ. 1992 - 01 - 01. - М.: Гос. комитет СССР по управлению качеством продукции и стандартам: Изд-во стандартов, 1992. $-14 \mathrm{c}$.

3. ГОСТ 4. 401 - 88. Автомобили грузовые. Номенклатура показателей. - Взамен ГОСТ $4.401-85$; введ. 1989 - 01 - 01. - М.: Гос. комитет СССР по стандартам: Изд-во стандартов, 1989. - 10 с.

4. ГОСТ Р 52280 - 2004. Автомобили грузовые. Общие технические требования. - Введ. 2007 - 01 01. - М.: Федеральное агентство по техническому регулированию и метрологии: ИПК Изд-во стандартов, 2005. - III, 9 c.

5. Кравец, В. Н. Измерители эксплуатационных свойств автотранспортных средств: учеб. пособие / В.Н. Кравец. - Н. Новгород: НГТУ им. Р.Е. Алексеева, 2014. - 157 с. 
6. Тарасик, В.П. Теория движения автомобиля: учеб. для вузов / В.П. Тарасик. - СПб.: БХВ - Петербург, 2006. -478 с.

7. Ларин, В.В. Теория движения полноприводных колёсных машин: учеб. / В.В. Ларин. - М.: МГТУ им. Н.Э. Баумана, 2010. - 391 с.

8. Кравец, В.Н. Теория автомобиля: учеб. для вузов / В.Н. Кравец, В.В. Селифонов. - М.: ООО «Гринлайт+», 2011. $-884 \mathrm{c.}$

9. Автомобили: теория эксплуатационных свойств: учеб. для студентов учреждений высш. проф. образования / А.М. Иванов, А.Н. Нарбут. А.С. Паршин [и др.]; под ред. А.М. Иванова. - М.: ИЦ «Академия», 2013. - $176 \mathrm{c}$.

\title{
V.N. Kravets, R.A. Musarsky, A.V. Tumasov \\ INFLUENCE OF TRANSMISSION PARAMETERS ON THE INDICATORS OF THE TRAFFIC-SPEED PROPERTIES OF THE TRUCK CAR
}

\author{
Nizhny Novgorod state technical university n.a. R.E. Alexeev
}

Purpose: Development of methods for determining indicators of traction - speed properties and the establishment of dependencies between the values of the design parameters of the transmission and indicators of traction - speed properties of a truck.

Design/methodology/approach: Determination of indicators of traction-speed properties of the studied truck is made on the basis of solving the equation of its rectilinear motion by graphical method with the construction of graphs of force and power balances, dynamic characteristics, accelerations, time and way of speed up of the car.

Findings: The influence of the number of gearbox steps and the transmission number of the main gear on the main indicators of traction-speed properties of the truck is shown.

Research limitations/implications: Using a graphical method to solve the equation of rectilinear motion of the car required a large amount of computational work and construction of 48 graphs, but allowed to obtain reliable results due to the use of real characteristics and parameters of the vehicle.

Originality/value: The originality of the results is due to the fact that the object of the research is a new model of the family of medium - tonnage vehicles of the GAZ group. The study will allow the company to more reasonably approach the choice of parameters for the transmission of the car GAZon Next 10.

Key words: truck, car transmission, manual speed gearbox, main gear, traction and speed properties of the car, indicators of traction and speed properties. 